\title{
Chicken or Egg: Which Came First?
}

\author{
Nayana George $^{\mathrm{a}}$, Benjamin Tharian ${ }^{\mathrm{b}}$, Sangeetha Tharian ${ }^{\mathrm{c}}$, Benny Paul ${ }^{\mathrm{d}, \mathrm{f}}$, Valsamma Chacko ${ }^{\mathrm{e}}$
}

\begin{abstract}
Cerebral venous sinus thrombosis (CVST) is quite an unusual diagnosis in pregnancy and puerperium. It can present with a wide range of symptoms and signs, and a high index of suspicion is required to diagnose this disorder. The diagnosis is challenging and could if left undiagnosed, result in life-threatening complications. Yet its management is simple with excellent prognosis, with an early diagnosis and timely intervention. We report a case of CVST in a young pregnant patient who presented with features of raised intracranial tension and loss of consciousness, after several weeks of hyperemesis gravidarum. She was treated with intravenous fluid resuscitation, low molecular weight heparin, steroids, and anti-cerebral edema measures. She subsequently made excellent recovery and is under follow-up.
\end{abstract}

Keywords: Cerebral venous sinus thrombosis; Hyperemesis gravidarum; Magnetic resonance venogram; Pregnancy complications

\section{Introduction}

Cerebral venous sinus thrombosis (CVST) is a unique condition, which is rarely seen with a highly variable clinical presentation and diverse etiologies. Though its actual incidence is not exactly known, it is estimated to be $5 / 1,000,000$. These account for $15-20 \%$ of young strokes. The condition of "occlusion of veins that drain" was first published in 1820 s and the

Manuscript accepted for publication October 29, 2014

aDepartment of Medicine, Western Hospital, Melbourne, Australia

bepartment of Medicine, NWAHS, Australia

${ }^{\mathrm{c} D e p a r t m e n t}$ of Obstetrics and Gynaecology, Cochin Medical College, Ernakulam, Kerala, India

${ }^{\mathrm{d} D e p a r t m e n t}$ of CVTS, Amrita Institute of Medical Sciences, Ernakulam, Kerala, India

eDepartment of Obstetrics and Gynaecology, Kerala Institute of Medical Sciences, Trivandrum, Kerala, India

${ }^{f}$ Corresponding Author: Benny Paul, Department of CVTS, AIMS, Ponekkara,

Ernakulam, Kerala 682041, India. Email: bpaulmenachery@yahoo.com

doi: http://dx.doi.org/10.14740/jnr294w first case of dural sinus thrombosis described by Ribes from France. The knowledge of the subject has grown tremendously since the original review by Tonelle et al in 1829. Sir Charles Symonds was the first to describe the association with benign intracranial hypertension as "otitic hydrocephalus". There are several possible etiologies though idiopathic in up to $30 \%$ of the patients. CVST is quite an unusual diagnosis in pregnancy and puerperium. It can present with a wide range of symptoms and signs, and a high index of suspicion is required to diagnose this disorder. It is often confused with eclampsia, especially if it develops on a background of preeclampsia. The diagnosis of CVST is challenging and could if left undiagnosed, result in life-threatening complications. Yet its management is simple with excellent prognosis, with an early diagnosis and timely intervention. In this paper, we aim to present a case of sinus thrombosis as a rare complication of an undertreated hyperemesis gravidarum (HG), with a good outcome with aggressive medical treatment.

\section{Case Report}

A 26-year-old primigravida presented in the 10th week of pregnancy, admitted obtunded with hemiparesis and delirium in the context of HG for which she was undergoing treatment in a local hospital. She did not have any significant past medical history and denied use of any over the counter medication/ herbal remedies or contraceptive pills.

On examination, the patient had bilateral papilledema, aphasia, right hemiplegia, in addition to being delirious, rapidly deteriorating to coma. There was no obvious nystagmus, anisocoria, neck stiffness or skin rash. Emergency magnetic resonance angiogram (MRA) and magnetic resonance venogram (MRV) showed extensive thrombosis involving superior/ inferior sagittal veins, bilateral transverse and left sigmoid sinuses (Fig. 1). There was in addition hemorrhagic infarct in bilateral fronto parietal, cortical and suboccipital regions with moderate mass effect and diffuse venous infarcts in the right frontal cortical and subcortical regions. Bloods showed minimal leucocytosis and prerenal failure (possibly from dehydration), hypokalemia (from hyperemesis) and minimally raised ESR. Vasculitic screen (including lupus anticoagulant) and thrombophilia screen were negative.

Patient was commenced on intravenous fluids, subcutane- 


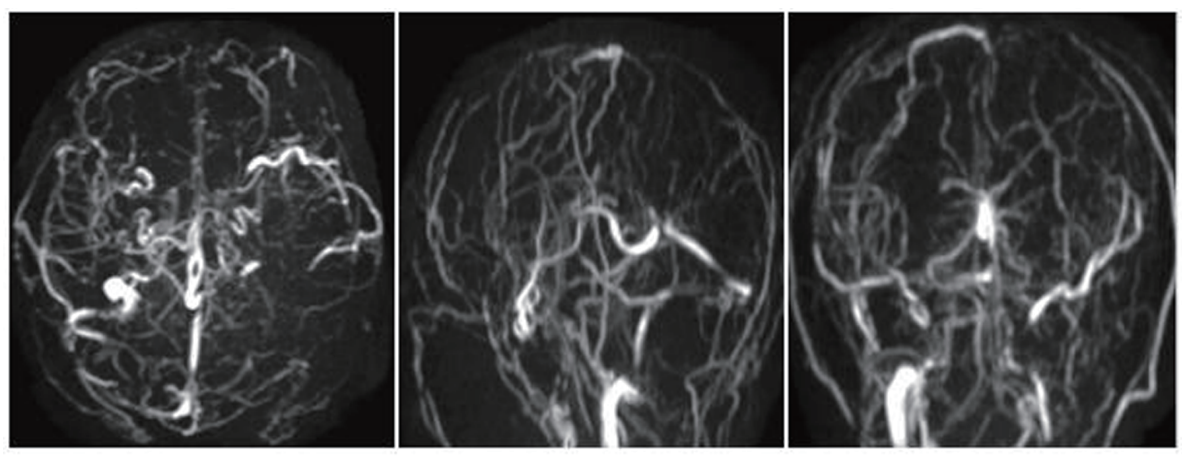

Figure 1. MRV before treatment.

ous enoxaparin twice daily, intravenous thiamine, levetiracetam, methyl prednisolone and anti-cerebral edema measures. Ultrasound of abdomen showed a 10 -week-old viable intrauterine pregnancy. She had an uneventful full term normal vaginal delivery. Her symptoms improved and she was discharged on day 9 with minimal neurologic deficit on enoxaparin (at her request instead of oral anticoagulant), acetazolamide (for 8 weeks), levetiracetam and outpatient physiotherapy and rehabilitation. Repeat MRI of brain in May 2012 showed encephalomalacia in left parietal and right parietoocciptial regions and no evidence of sinus thrombosis (Fig. 2).

\section{Discussion}

New onset of a neurologic syndrome in pregnancy could be the manifestation of flare up of a preexisting illness, initial presentation of a disease unique to this group or a pregnancy unrelated condition [1]. The various neurologic conditions occurring in pregnancy and peripartum period include eclampsia (specific to pregnancy), ischemic stroke, intracerebral hemorrhage, post partum angiopathy, posterior reversible encephalopathy syndrome (PRES), Wernicke-Korsakoff's syndrome (WKS), amniotic fluid embolism, pituitary apoplexy and CVST [1]. The latter is a well-recognized, though uncommon diagnosis in pregnancy and puerperium, with an estimated incidence of $5 / 1,000,000$ [2]. CVST if left undiagnosed could result in lifethreatening complications [3]. The mortality associated with CVST is 7\% [4]. Therefore, a high index of suspicion is required to diagnose this disorder. The clinical presentation of CVST can vary widely from severe headache to deep coma. The most common presentation is headache in $97 \%$ with only $10 \%$ presenting as a thunder clap headache [1]. It can also present as seizure attacks (47\%) and paresis (43\%) [5]. Nonspecific symptoms include nausea and lethargy. Convulsions, often focal seizures and loss of consciousness, papilledema, intracranial bleeding and internal cerebral vein occlusion are poor prognostic markers [6]. Women in the age group of $25-$ 35 years are commonly affected and it occurs more often during puerperium than pregnancy [6]. It has been increasingly recognized in the first trimester possibly due to better management of thrombophilias leading to higher pregnancy rates [7]. It is more common in lower socioeconomic regions due to delayed presentation often due to lack of awareness among public and health care professionals and poor health care access, malnutrition and increasing infections $[8,9]$.

Virchow's triad of predisposing factors to thrombosis, including hypercoagulability, stasis and loss of endothelial integrity still holds true. Though there are more than 100 causes of CVST, even with extensive investigation, no cause is identified in $20-25 \%$ of patients [10]. The common causes are hypercoagulable states such as pregnancy and puerperium, the use of oral contraceptives, malignancy, dehydration, obesity, vasculitides, intracranial infections and Behcets disease [3]. Women using oral contraceptives have an increased risk of CVST by approximately $20 \%$ [9]. Ten percent to $15 \%$ of CVST are due to inherited prothrombotic tendencies such as factor $\mathrm{V}$ Leidan mutation, protein $\mathrm{C}$ and $\mathrm{S}$ and anti-thrombin III deficiencies. Infections of head, face and middle ear leading to CVST are less common because of modern broad spectrum antibiotic treatment [11].

$\mathrm{HG}$ is a severe form of morning sickness, with a multifactorial neurohormonal basis, affecting 1:200 pregnancies and is the most common indication for hospital admission during the first trimester [12]. HG as a cause of cortical sinus thrombosis has been reported only once in literature [13]. CVST occurring in the first trimester is rare with mortality as high as $30 \%$ and $20 \%$ having residual weakness. The mechanism includes severe dehydration contributing to hyperviscosity that aggravates the physiologically hypercoagulable milieu (secondary to hyperestrogenism) $[13,14]$. The other risk factors include cesarean section, traumatic delivery, anemia, hyper homocysteinemia and CSF hypotension secondary to leak following an epidural anesthesia. The diagnosis of CVST in this setting is often delayed due to symptoms of raised intracranial tension being attributed to hyperemesis, until focal neurologic deficit sets in. The main differential for an acute neurological complication in the setting of $\mathrm{HG}$ is WKS that could also present with coma in severe cases [14]. The triad of ataxia, confusion and ophthalmoplegia and response to IV thiamine should confirm WKS [15-17]. The condition is due to poor nutritional intake, hyperemesis and increased metabolic demand $[15,18]$.

Neuroimaging remains the gold standard investigation [4, $9,11,19,20]$. A CT scan is useful for the diagnosis of infarcts or hemorrhages and to rule out other intracranial pathology, but its results can be entirely normal [20]. The most sensitive 


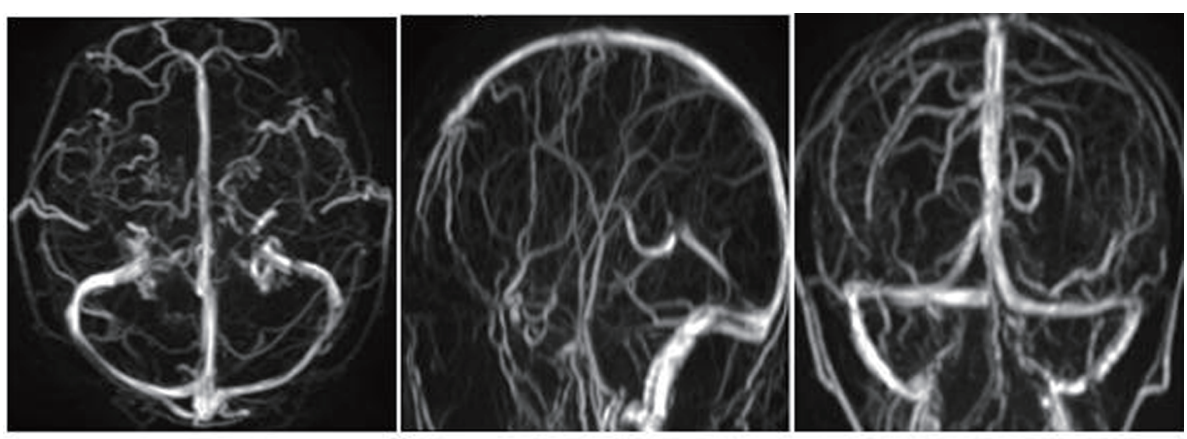

Figure 2. MRV after treatment.

imaging modality is MRV especially with gradient spin-echo sequence [21]. The combination of abnormal signal and a corresponding absence of flow in a cerebral venous sinus is suggestive of CVST [22]. MRI in WKS would show symmetrical increase in signal intensity in the mesencephalic tegmentum, mamillary body and medial thalamus $[15,17]$. Since the diagnosis cannot be made with certainty until MR, such neurologic syndromes should be treated with intravenous hydration (to reduce viscosity and progressive thrombosis), especially if there is clinical and biochemical evidence of dehydration from the hyperemesis, in addition to intravenous thiamine, the treatment of choice for potential WKS. Treatment modalities for CVST are anticoagulation, thrombolytic therapy and in some cases surgical thrombectomy $[9,10]$. Anticoagulation with low molecular weight heparin is the treatment of choice followed by oral warfarin for a 6 - 12 months period with repeat imaging to establish the status of recanalization. There are no randomized trials giving us management guidelines. In our patient warfarin was avoided as she was pregnant and then discharged on enoxaparin at her request. Prognosis is quite variable though better than CVST occurring outside of pregnancy and in men due to unknown factors. The outcome can vary from complete recovery to death; however, prospective studies have reported independent survival rates of approximately 80\% [23]. Mortality is primarily due to intracerebral hemorrhage. The risk of recurrent CVST in future pregnancies and puerperium is low [24].

In conclusion, CVST may present with a wide range of symptoms and signs. Diagnosis can be confirmed by MRV in most cases. It is a life-threatening condition if left undiagnosed, but remains a potentially reversible neurologic emergency if managed timely and appropriately. It should be considered in every woman with neurological symptoms in pregnancy and puerperium. Early diagnosis and timely intervention can save lives and prevent debilitating consequences. This case also highlights the need for aggressive fluid replacement in patients with $\mathrm{HG}$, often intravenous and potentially needing parenteral nutrition, to prevent devastating neurologic sequelae in this select group of pregnant women and thus minimize the morbidity and mortality associated with the condition. The awareness has to be raised amongst midwives, obstetricians, emergency physicians and the general public alike since we strongly believe the egg came first and not the chicken. Such patients should be cared for in a center that has the facility for multidisciplinary input, involving neurologists, neurosurgeons, interventional radiologists, high risk obstetricians and critical care in addition to dedicated neurorehabilitation. With improved public and medical awareness of the condition, increasing availability and accessibility to MRI and ongoing research in the field leading to better understanding, the outcomes would improve further.

\section{Conflict of Interest}

No conflict of interest was reported.

\section{Grant Support}

No grants or financial support was accepted for this article.

\section{References}

1. Edlow JA, Caplan LR, O'Brien K, Tibbles CD. Diagnosis of acute neurological emergencies in pregnant and postpartum women. Lancet Neurol. 2013;12(2):175-185.

2. Bousser MG, Ferro JM. Cerebral venous thrombosis: an update. Lancet Neurol. 2007;6(2):162-170.

3. Soydinc HE, Ozler A, Evsen MS, Sak ME, Turgut A, Basaranoglu S, Dusak A, et al. A case of cerebral sinus venous thrombosis resulting in mortality in severe preeclamptic pregnant woman. Case Rep Obstet Gynecol. 2013;2013:402601.

4. Ferro JM, Lopes MG, Rosas MJ, Ferro MA, Fontes J. Long-term prognosis of cerebral vein and dural sinus thrombosis. results of the VENOPORT study. Cerebrovasc Dis. 2002;13(4):272-278.

5. de Bruijn SF, de Haan RJ, Stam J. Clinical features and prognostic factors of cerebral venous sinus thrombosis in a prospective series of 59 patients. For The Cerebral Venous Sinus Thrombosis Study Group. J Neurol Neurosurg Psychiatry. 2001;70(1):105-108.

6. Cantu C, Barinagarrementeria F. Cerebral venous thrombosis associated with pregnancy and puerperium. Review of 67 cases. Stroke. 1993;24(12):1880-1884.

7. Cantu-Brito C, Arauz A, Aburto Y, Barinagarrementeria F, Ruiz-Sandoval JL, Baizabal-Carvallo JF. Cerebrovas- 
cular complications during pregnancy and postpartum: clinical and prognosis observations in 240 Hispanic women. Eur J Neurol. 2011;18(6):819-825.

8. Mas JL, Lamy C. Stroke in pregnancy and the puerperium. J Neurol. 1998;245(6-7):305-313.

9. Martinelli I. Cerebral vein thrombosis. Thromb Res. 2013;131(Suppl 1):S51-54.

10. Lewis MB, Bousser MG. Cerebral venous thrombosis: nothing, heparin, or local thrombolysis? Stroke. 1999;30(8):1729.

11. Deschiens MA, Conard J, Horellou MH, Ameri A, Preter M, Chedru F, Samama MM, et al. Coagulation studies, factor V Leiden, and anticardiolipin antibodies in 40 cases of cerebral venous thrombosis. Stroke. 1996;27(10):17241730 .

12. Loh KY, Sivalingam N. Understanding hyperemesis gravidarum. Med J Malaysia. 2005;60(3):394-399; quiz 400 .

13. Kennelly MM, Baker MR, Birchall D, Hanley JP, Turnbull DM, Loughney AD. Hyperemesis gravidarum and first trimester sagittal sinus thrombosis. J Obstet Gynaecol. 2008;28(4):453-454.

14. Zara G, Codemo V, Palmieri A, Schiff S, Cagnin A, Citton V, Manara R. Neurological complications in hyperemesis gravidarum. Neurol Sci. 2012;33(1):133-135.

15. Chiossi G, Neri I, Cavazzuti M, Basso G, Facchinetti F. Hyperemesis gravidarum complicated by Wernicke encephalopathy: background, case report, and review of the literature. Obstet Gynecol Surv. 2006;61(4):255-268.

16. Selitsky T, Chandra P, Schiavello HJ. Wernicke's enceph- alopathy with hyperemesis and ketoacidosis. Obstet Gynecol. 2006;107(2 Pt 2):486-490.

17. Sulaiman W, Othman A, Mohamad M, Salleh HR, Mushahar L. Wernicke's Encephalopthy Associated with Hyperemesis Gravidarum - A Case Report. Malays J Med Sci. 2002;9(2):43-46.

18. Eboue C, Carlier-Guerin C, de La Sayette V, Grall JY, Herlicoviez M. [A rare complication of vomiting in pregnancy: Wernicke's encephalopathy]. J Gynecol Obstet Biol Reprod (Paris). 2006;35(8 Pt 1):822-825.

19. Coutinho JM, Gerritsma JJ, Zuurbier SM, Stam J. Isolated cortical vein thrombosis: systematic review of case reports and case series. Stroke. 2014;45(6):1836-1838.

20. Sagduyu A, Sirin H, Mulayim S, Bademkiran F, Yunten N, Kitis O, Calli C, et al. Cerebral cortical and deep venous thrombosis without sinus thrombosis: clinical MRI correlates. Acta Neurol Scand. 2006;114(4):254-260.

21. Fink JN, McAuley DL. Cerebral venous sinus thrombosis: a diagnostic challenge. Intern Med J. 2001;31(7):384390.

22. Ayanzen RH, Bird CR, Keller PJ, McCully FJ, Theobald MR, Heiserman JE. Cerebral MR venography: normal anatomy and potential diagnostic pitfalls. AJNR Am J Neuroradiol. 2000;21(1):74-78.

23. Masuhr F, Mehraein S, Einhaupl K. Cerebral venous and sinus thrombosis. J Neurol. 2004;251(1):11-23.

24. Mehraein S, Ortwein H, Busch M, Weih M, Einhaupl K, Masuhr F. Risk of recurrence of cerebral venous and sinus thrombosis during subsequent pregnancy and puerperium. J Neurol Neurosurg Psychiatry. 2003;74(6):814-816. 